\title{
Effect of sample bias on backscattered ion spectroscopy in the helium ion microscope
}

\author{
G. Behan, J. F. Feng, and H. Z. Zhang ${ }^{\text {a) }}$ \\ School of Physics and Centre for Research on Adaptive Nanostructures and Nanodevices (CRANN), Trinity \\ College Dublin, Dublin 2, Republic of Ireland \\ P. N. Nirmalraj and J. J. Boland \\ School of Chemistry and Centre for Research on Adaptive Nanostructures and Nanodevices (CRANN), \\ Trinity College Dublin, Dublin 2, Republic of Ireland
}

(Received 11 June 2010; accepted 20 September 2010; published 20 October 2010)

\begin{abstract}
The authors present experimental results showing the effect of an applied bias voltage on backscattered ion spectra acquired from thin films of ruthenium and hafnia in the helium ion microscope. A characteristic peak associated with the presence of a thin layer of material is observed to shift as a function of sample bias voltage. The magnitude of this shift is measured, and the authors qualitatively estimate the composition of their samples as well as investigate the neutralization of ions by the sample in the helium ion micoscope (HeIM). They discuss the phenomenona in terms of thin films of ruthenium and hafnia and show the implications of these results on HeIM spectroscopy. (C) 2010 American Vacuum Society. [DOI: 10.1116/1.3502667]
\end{abstract}

\section{INTRODUCTION}

The helium ion micoscope (HelM) is a new tool for the high-resolution imaging of surfaces. ${ }^{1}$ In comparison to the more common scanning electron microscope (SEM), the HelM offers a smaller probe size and a larger secondary electron yield, resulting in images with richer contrast and finer surface detail. ${ }^{2}$ While the analytical capability of the SEM is generally realized by the attachment of an energy dispersive $\mathrm{x}$-ray spectrometer, the energy of the primary ion beam in the HelM is typically too low $(\approx 30 \mathrm{keV})$ to generate characteristic $\mathrm{x}$ rays. ${ }^{3}$ It is possible, however, to collect the backscattered helium ions and measure their energy to produce a spectrum. Such spectra can indicate both the thickness and composition of a specimen as well as give a depth profile of the sample. The principle behind this technique is the same as Rutherford backscattered spectroscopy (RBS), with the exception that the ion energies used are many times smaller than the typical $2 \mathrm{MeV}$ employed in RBS experiments. ${ }^{4}$ One advantage of using the HelM is that unlike RBS, we are able to image the sample and acquire analytical information simultaneously. Moreover, the size of the HelM probe is typically less than $1 \mathrm{~nm}$; thus, the signal is gathered from a much smaller area than the micron sized area achieved in micro-RBS. ${ }^{5}$ As a result, the HelM shows some promise as a subnanometer imaging and analytical instrument for the characterization of nanostructures and thin films. ${ }^{6}$

On an ion's approach toward the sample surface, there is a high probability that the incident helium ion will be neutralized, ${ }^{7,8}$ and therefore a significant proportion of the backscattered flux are neutral particles. Neutrals and ions exhibit the same efficiency to our solid-state detector, ${ }^{6}$ and it is unable to discriminate between the signal generated from

${ }^{a)}$ Electronic mail: hongzhou.zhang@tcd.ie either particle. If we consider an ion with energy $E_{0}$ incident on a thin layer of material, the backscattered energy is $E_{1}$ $=k E_{0}$, where $k$ is the kinematic factor. The constant $k$ is dependent on the mass of the incident ion, the scattering angle, and the mass of the target atom, $M_{1}, \theta$, and $M_{2}$, respectively, and can be calculated from the following equation: ${ }^{4}$

$$
k=\left[\frac{\left(M_{2}^{2}-M_{1}^{2} \sin ^{2} \theta\right)^{1 / 2}+M_{1} \cos \theta}{M_{1}+M_{2}}\right]^{2} .
$$

Thus, Eq. (1) provides a qualitative way of estimating the composition of the material by calculating the mass of the target atom. This approach is used in simulation packages such as SIMNRA (Ref. 9) to provide an initial estimate of the position of any characteristic peaks present in the spectra.

The above treatment assumes that any potential at the sample's surface is negligible. In fact, the energies of the detected ions and neutrals are dependent not only on the scattering process, but also on the potential experienced by the particles near the sample surface. ${ }^{10}$ For a potential, $V$, the backscattered ion energy is modified and becomes

$$
E_{1}=k\left(E_{0}-q_{1} V\right)+q_{2} V
$$

where $q_{1}$ and $q_{2}$ are the charge states of the incoming and outgoing particles, respectively. For singly ionized helium $q=1$, while in the case of a neutral $q=0$. The local electric potential of the sample can therefore influence the measurement by effectively changing the energy of the incident ion.

There are two ways in which the sample can be raised to a particular potential. The first involves the fact of any charged beam microscopy that if the sample is insufficiently grounded, a charge can build up over time due to the bombardment of the sample with the primary charged particles, positive helium ions in this instance. ${ }^{11}$ Second, to enhance the contrast of secondary electron imaging, it is common to apply a potential to the sample through the stage. The latter 


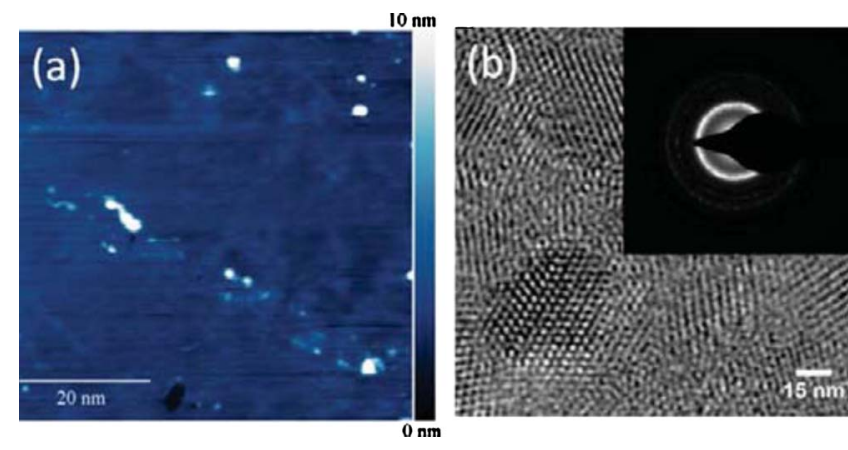

FIG. 1. (Color online) AFM image of ruthenium deposited on a silicon substrate. The surface roughness is estimated to be $2.5 \pm 0.8 \mathrm{~nm}$ based on an average of several topographic images. A number of surface adsorbates also appear to be present on the surface of the film. (b) TEM image of the ruthenium film deposited onto an amorphous carbon film. (Inset) Diffraction pattern taken from the ruthenium film; the ring pattern confirms the polycrystallinity of the film. From the width of the ring, the average grain size is $\approx 12.5 \mathrm{~nm}$.

method is employed in these experiments for demonstrative purposes. Without an applied bias, no evidence of charging was observed during imaging.

The variation in the surface potential and the effect this has on the detection of backscattered particles impact the reliability and accuracy of the spectrometry. It is therefore critical to understand the effect for the further development and application of the HelM as an analytical tool. In this article, we investigate the shift in the characteristic thin-film peak as a function of sample bias voltage for two materials.

\section{EXPERIMENTAL METHODS}

All backscattered ion spectra were acquired on a Carl Zeiss Orion Plus HelM equipped with a modified solid-state detector $^{6}$ that has an energy resolution of $\approx 5 \mathrm{keV}$. The spectrometer was calibrated before the experiment to ensure the accuracy of the measurements using the hafnia standard sample. Two thin-film samples were prepared. The first, a 2 $\mathrm{nm}$ ruthenium film, was dc-magnetron sputtered onto a silicon substrate at room temperature by a Shamrock deposition tool with a base pressure of $8.0 \times 10^{-8}$ Torr using highpurity argon. The deposition rate of the tool was calibrated before deposition and measured to be $0.92 \mathrm{~nm} / \mathrm{min}$; therefore, the deposition time was chosen to be $2.17 \mathrm{~min}$. To facilitate later transmission electron microscopy (TEM) (FEI Titan, $300 \mathrm{kV}$ ) analysis, the metal was also deposited onto an amorphous carbon film. An atomic force microscope (AFM) (Veeco Systems, Dimension V) was later used to estimate surface roughness for the ruthenium sample [see Fig. 1(a)]. The second sample was a film of hafnia, nominally $2 \mathrm{~nm}$ thick, on a silicon substrate purchased from Geller Microanalytical. ${ }^{12}$ Both films have a native interfacial layer of silica present between the metallic layer and the substrate.

The two thin-film samples were mounted on SEM stubs, and the samples were electrically grounded (see Fig. 2 for an image of the hafnia film). A sample bias voltage was applied through the sample stage and varied between -500 and $+500 \mathrm{~V}$. A spectrum was acquired at every $100 \mathrm{~V}$ increment.
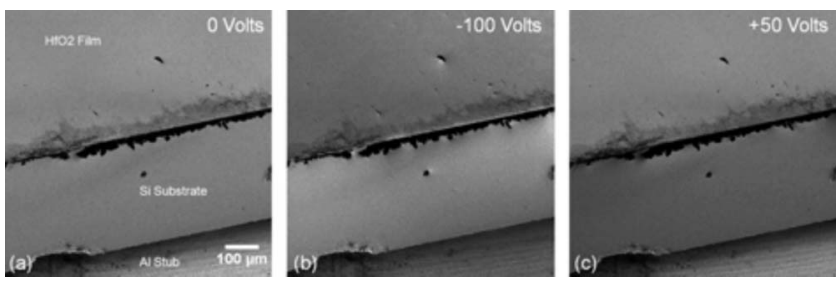

FIG. 2. Secondary electron images of a hafnia film deposited on a silicon substrate at (a) $0 \mathrm{~V}$, (b) $-100 \mathrm{~V}$, and $+50 \mathrm{~V}$ sample biases acquired using the HeIM. The area for spectrum acquisition was $100 \mu \mathrm{m}^{2}$, and the film was not tilted. The secondary electron contrast is observed to vary with the incident energy of the ions.

During spectrum acquisition, the dwell time was $2 \mu$ s, and a live-time of $200 \mathrm{~s}$ was used to improve the signal-to-noise ratio. The angle of incidence was $0^{\circ}$ from the sample normal, and the scattering angle into the detector was $115^{\circ}$. The energy of the primary helium incident ions was $30.8 \mathrm{keV}$ with an energy spread of $20 \mathrm{meV}$, and the beam current was kept at around $17.8 \pm 0.1 \mathrm{pA}$. A high beam current provides a large signal-to-noise ratio and simplifies the curve fitting and data analysis. The signal was gathered from a region of $100 \mu \mathrm{m}^{2}$ in an area from each sample. To minimize any electrical interference, all other detectors were switched off for the duration of the experiment and the sample was randomly moved after each spectrum was recorded to reduce any buildup of surface contamination.

It is the position of the thin-film peak that is of primary concern for our measurements, and the values are determined using the curve fitting routines found in the ORIGIN (Ref. 13) plotting software. This software employs the LevenbergMarquardt algorithm ${ }^{14}$ to find the best fit to the experimental data. The algorithm fitted the region between the full-width at half-maximum points of the thin-film peak. The quality of each fit was reported to be $>0.95$ where a value of 1 would indicate an exact fit.

\section{RESULTS AND DISCUSSION}

The ruthenium and hafnia films uniformly cover the substrate, and Fig. 1(a) is an AFM image of the ruthenium film's surface, indicating the surface roughness. Based on several such topographic images, the surface roughness of the ruthenium film is calculated to be $2.5 \pm 0.8 \mathrm{~nm}$. Surface roughness can result in the change of the shape of the peak and can influence the film thickness measurement although it is more significant in the case of the grazing angles of the incident or emerging ions. ${ }^{15}$ It should be noted that despite this variation in surface roughness, the deposited layers can still be considered thin, and Eq. (2) remains valid for our analysis. A transmission electron microscopy investigation of the ruthenium film deposited on the carbon demonstrated that the film is polycrystalline in nature [see Fig. 1(b)]. According to the specifications of the hafnium dioxide thin-film sample, it is also polycrystalline. ${ }^{12}$ From fitting the zero biased spectra of the two samples in SIMNRA, the film thicknesses are estimated to be $2.35 \pm 0.4 \mathrm{~nm}$ for hafnia and $2.88 \pm 0.5 \mathrm{~nm}$ for ruthenium. 

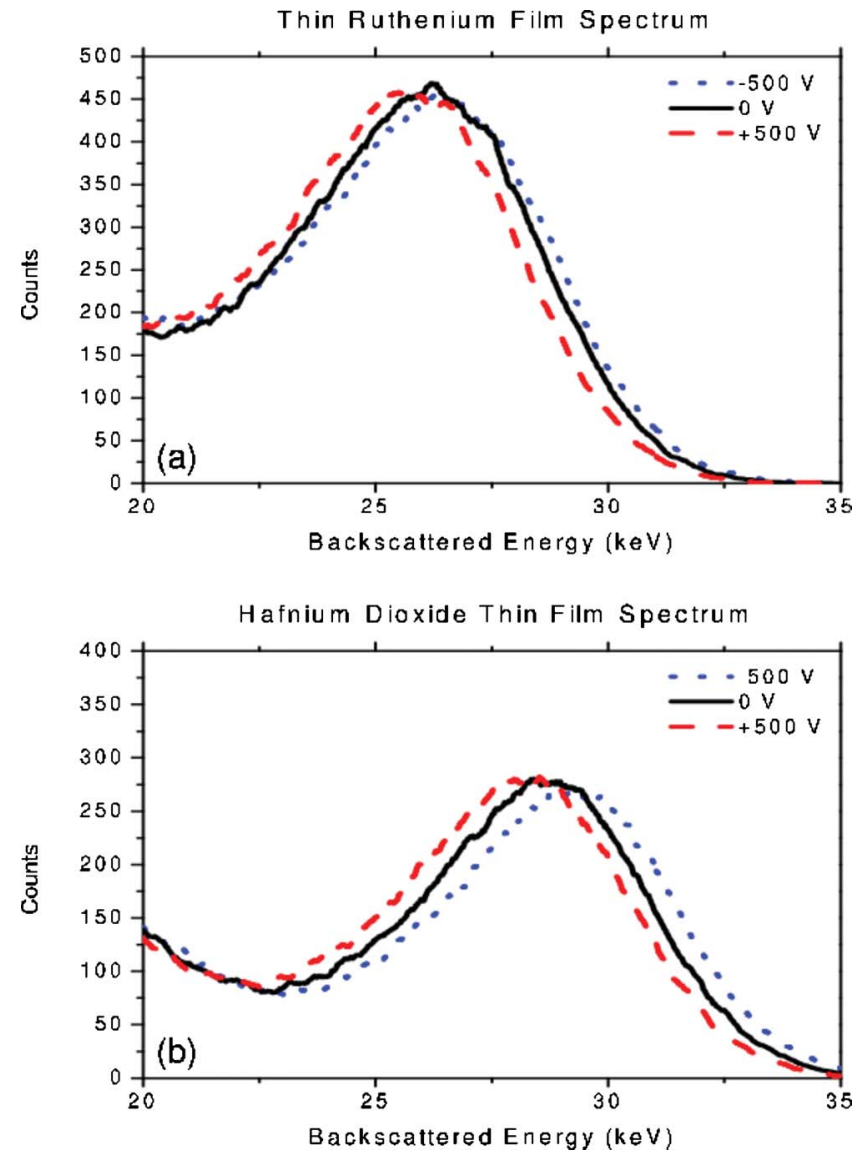

FIG. 3. (Color online) Backscattered energy spectra from thin films of (a) ruthenium and (b) hafnia at different sample bias voltages. A systematic shift in the peak position is apparent in the case of both samples. The data have been smoothed using a moving-average filter with a $300 \mathrm{eV}$ width for presentation purposes.

Two spectra acquired from the samples at different sample bias voltages are shown in Fig. 3. In this figure, it is clear that there is a noticeable shift in the peak position compared to the unbiased case (the solid lines in Fig. 3). With an applied positive sample bias (the dashed lines in Fig. 3), there is a shift in the peak toward lower energies, while a negative sample bias results in a shift toward higher energies (the dotted lines in Fig. 3). The peak shift as a function of the sample bias voltage, as determined by fitting a Gaussian to the peak, is plotted in Fig. 4 along with the best fit lines. The slopes of the best fit lines for the ruthenium and hafnium dioxide samples are 0.821 and 0.913 , respectively. If we now consider the magnitude of the peak shift, $\Delta E_{1}$, by explicitly writing out the formula for the peak position using Eq. (2),

$$
\begin{aligned}
& E_{1}=k E_{0}, \\
& E_{1}^{\prime}=k E_{0}-q_{1} k V+q_{2} V, \\
& \Delta E_{1}=E_{1}^{\prime}-E_{1}=\left(-q_{1} k+q_{2}\right) V .
\end{aligned}
$$

From Eq. (3), it is apparent that the peak shift is proportional to the sample bias $V$, and the slope of the linear relation is given by $-q_{1} k+q_{2}$ as long as the charge state of the
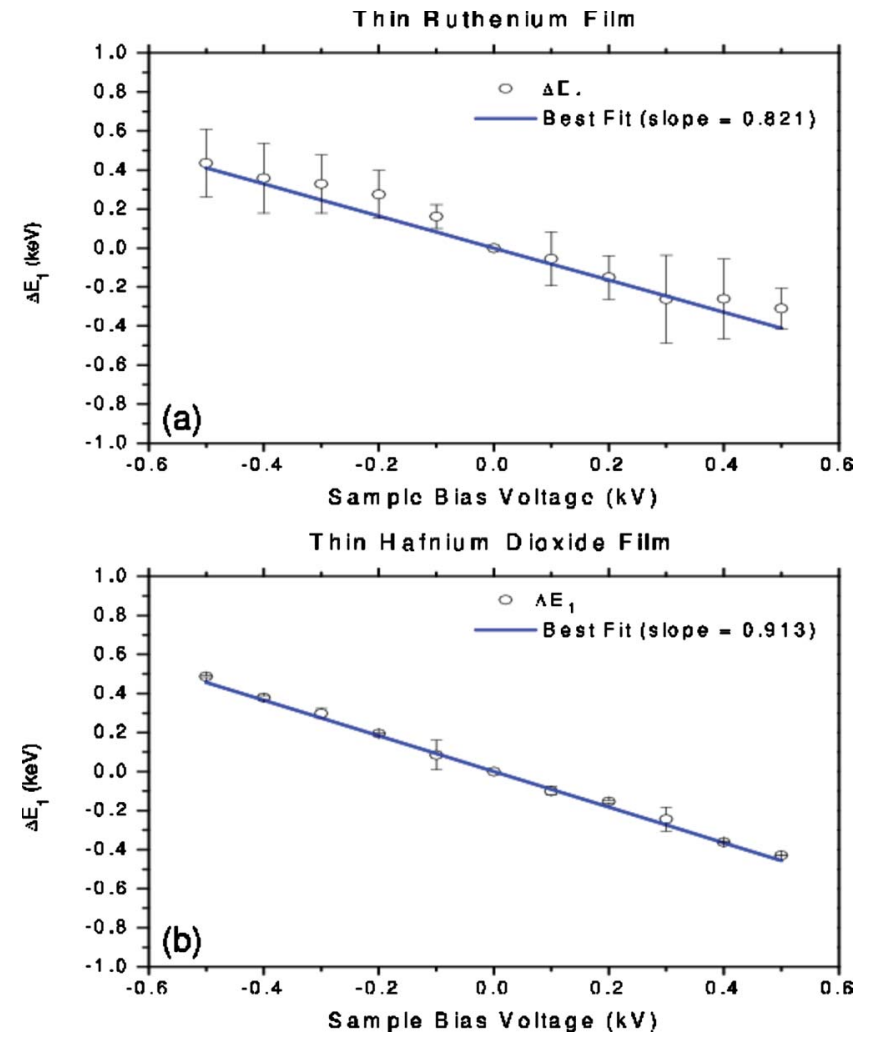

FIG. 4. (Color online) Measured peak position and the best fit lines for (a) ruthenium and (b) hafnium dioxide. The measured slopes and, therefore, the values of $k$ are found to be 0.821 and 0.913 , respectively.

scattered particle, $q_{2}$, does not vary with the sample bias $V$ for the primary ion. The kinematic factor, $k$, can therefore be extracted from the measured slope for a given set of $q_{1}$ and $q_{2} .{ }^{16}$ If we assume that the majority of detected particles are neutrals, where $q_{1}=1$ and $q_{2}=0$, the kinematic factor is equal to the measured slope. From our experimental configuration, the calculated kinematic factors [see Eq. (1)] are 0.893 and 0.938 for ruthenium and hafnium, respectively. ${ }^{9}$ The kinematic factors measured for both samples are in reasonable agreement with the calculated values with an error of $8 \%$ for ruthenium and $3 \%$ for the hafnium dioxide film. However, we should note that at large bias voltages the ruthenium measurements appear to cease behaving linearly and the deviation increases while the hafnium dioxide data remain linear.

For a possible explanation of this nonlinear phenomenon, we need to consider the assumption we previously madethat neutrals dominate the number of detected particles. Since the rate of neutralization at the sample's surface depends on the ion energy and the respective weighting of the ion and neutral peaks in the measured sum peak can vary, the ion's landing energy is modified by the sample bias. For a set combination of ion, target, and beam energy, the probability $P$ of the incident ion retaining its charge on approach to the sample tends to obey the following rule: ${ }^{4,7}$

$$
P=\exp \left(-v_{c} / v_{\perp}\right),
$$

where $v_{c}$ is a characteristic velocity of the projectile-target system and $v_{\perp}$ is the component of the incident ion's veloc- 
ity perpendicular to the sample surface. The value of $v_{c}$ is dependent on both the type of incident ion and the target material. For a constant, $v_{c}$, the probability of ion neutralization decreases drastically as $v_{\perp}=\sqrt{2\left(E_{0}-e V\right) / m}$ increases. In terms of our experiment, this would correspond to increasing the negative bias voltage on the sample where from Fig. 4 the measured position begins to deviate noticeably from a linear behavior. Over the range of velocities used in this experiment, the probability can vary by as much as $10 \%$. Essentially, we cannot distinguish between the neutral and ion contributions to the backscattered peak within the bias voltage range and energy resolution available to us on our microscope. While the neutral peak can shift [see (Eq. (2))] as a linear function of the bias voltage, it is the position of the sum peak that we measure which is influenced by the weight of the unresolved ion and neutral peaks.

Taking the backscattered ions into account, we define an effective charge state for the outgoing particles as $q_{2, \text { eff }}=(1$ $-P) q_{\text {neutral }}+(P) q_{\text {ion }}=P$, where $P$ is the ion survival probability $\left[\right.$ Eq. (4)], $q_{\text {neutral }}=0$, and $q_{\text {ion }}=1$. We can also estimate $q_{2, \text { eff }}=k-s$, where $k$ takes the theoretical value of the kinematic factor and $s$ is the measured slope from the curves in Fig. 4. If this value is of the same order of magnitude as our error, it would suggest, from Eq. (4), that the characteristic velocities are $\approx 10^{8} \mathrm{~cm} / \mathrm{s}$ for both samples. To the best of our knowledge, the values of $v_{c}$ do not appear in the literature for the samples used herein, and so it is difficult to provide a direct comparison with this approximation. A survey of various metallic films ${ }^{17-19}$ would indicate that $v_{c}$ tends to increase ${ }^{20}$ as a function of $v_{\perp}$ toward $>10^{8} \mathrm{~cm} / \mathrm{s}$. Therefore, our estimate appears to agree, in order of magnitude, with other such metallic samples.

Another possible cause for this difference may be due to the presence of surface adsorbates on the sample such as those shown in Fig. 1(a). Such adsorbates can modify the work function of the material and reduce the rate of ion neutralization. $^{21-23}$ As hydrocarbons are the most likely source of contaminant, their effect can be reduced if due care is taken before loading the sample such as plasma cleaning as was done here.

\section{CONCLUSIONS}

In conclusion, we observed an energy shift in the backscattered ion spectroscopy due to an applied sample bias voltage. Although the samples used in this work were not insulating, in principle this energy shift would also be present for an insulating sample, and this deviation from the theoretical model may confuse analytical results. A series of spectra taken at different bias voltages can still be used to determine the kinematic factor of the material by observing the shift of the characteristic peak, and from that the scattering element can be estimated. However, at an increasingly high bias voltage, a systematic correction factor may need to be introduced for a combination of ions and targets.

We have demonstrated these phenomena for two materials, hafnia and ruthenium, with good agreement with theory, and this method should be applicable to insulating samples where the peak will be observed to shift due to sample charging.

\section{ACKNOWLEDGMENTS}

The authors would like to acknowledge Markus Boese and Colm Faulkner at the CRANN Advanced Microscopy Laboratory and Mike Coey at Trinity College for their assistance and useful discussions. The work at the School of Physics and the Center for Research on Adaptive Nanostructures and Nanodevices at Trinity College Dublin is supported by the Science Foundation Ireland under Grant No. 07/SK/ I1220a. The TEM work was conducted under the framework of the INSPIRE program, funded by the Irish Government's Programme for Research in Third Level Institutions, Cycle 4, National Development Plan, Grant No. 2007-2013.

${ }^{1}$ J. Notte, R. Hill, S. McVey, L. Farkas, R. Percival, and B. Ward, Microsc. Microanal. 12, 126 (2006).

${ }^{2}$ D. C. Bell, Microsc. Microanal. 15, 147 (2009).

${ }^{3}$ D. C. Joy, H. M. Meyer, M. Bolorizadeh, Y. Lin, and D. Newbury, Scanning 29, 1 (2007).

${ }^{4}$ J. C. Vickerman and I. S. Gilmore, Surface Analysis: The Principal Techniques (Wiley, New York, 2009), p. 215

${ }^{5}$ K. Inoue, M. Takai, K. Matsunaga, M. Izumi, K. Gamo, S. Namba, and M. Satou, Nucl. Instrum. Methods Phys. Res. B 30, 580 (1988).

${ }^{6}$ S. Sijbrandij, J. Notte, B. Thompson, C. Huynh, C. Sanford, and L. Scipioni, Microsc. Microanal. 15, 220 (2009).

${ }^{7}$ H. D. Hagstrum, Phys. Rev. 96, 336 (1954).

${ }^{8}$ M. Yoshiaki and H. Teruo, Surf. Sci. 97, 283 (1980).

${ }^{9}$ M. Mayer, American Institute of Physics Conference Series No. 475, 1999 (unpublished), pp. 541-544.

${ }^{10}$ D.-E. Arafah, J. D. Meyer, H. Sharabati, and A. Mahmoud, Phys. Rev. A 39, 3836 (1989).

${ }^{11}$ L. Reimer, Scanning Electron Microscopy: Physics of Image Formation and Microanalysis, 2nd ed. (Springer, Berlin, 1998), p. 120.

${ }^{12}$ Geller Microanalytical, http://www.gellermicro.com/

${ }^{13}$ OriginLab, http://www.originlab.com/

${ }^{14}$ W. H. Press, S. A. Teukolsky, W. T. Vetterling, and B. P. Flannery, Numerical Recipes: The Art of Scientific Computing (Cambridge University Press, Cambridge, England, 2007), p. 683.

${ }^{15}$ R. D. Edge and U. Bill, Nucl. Instrum. Methods 168, 157 (1980).

${ }^{16}$ K. Takahiro, A. Terai, K. Kawatsura, H. Naramoto, S. Yamamoto, B. Tsuchiya, S. Nagata, and F. Nishiyama, Jpn. J. Appl. Phys., Part 1 45, 1823 (2006).

${ }^{17}$ M. Sasaki, P. J. Scanlon, S. Ermolov, and H. H. Brongersma, Nucl. Instrum. Methods Phys. Res. B 190, 127 (2002).

${ }^{18}$ T. Kravchuk, V. A. Esaulov, A. Hoffman, and R. C. Monreal, Nucl. Instrum. Methods Phys. Res. B 232, 27 (2005).

${ }^{19}$ A. A. Almulhem, Vacuum 81, 150 (2006).

${ }^{20}$ E. C. Goldberg, R. Monreal, F. Flores, H. H. Brongersma, and P. Bauer, Surf. Sci. 440, L875 (1999).

${ }^{21}$ M. Beckschulte and E. Taglauer, Nucl. Instrum. Methods Phys. Res. B 78, 29 (1993).

${ }^{22}$ R. Cortenraad, A. W. Denier van der Gon, H. H. Brongersma, S. N. Ermolov, and V. G. Glebovsky, Surf. Interface Anal. 31, 200 (2001).

${ }^{23}$ Y. Yang and J. A. Yarmoff, J. Vac. Sci. Technol. 21, 1317 (2003). 